\title{
Towards the design of efficient nonbeacon-enabled ZigBee networks
}

\author{
Helena Fernández-López ${ }^{\mathrm{a}, \mathrm{b}, *}$, José A. Afonso ${ }^{\mathrm{a}, \mathrm{b}}$, J.H. Correia ${ }^{\mathrm{a}, \mathrm{b}}$, Ricardo Simoes ${ }^{\mathrm{c}, \mathrm{d}, \mathrm{e}}$ \\ a Department of Industrial Electronics, University of Minho, 4800-058 Guimarães, Portugal \\ ${ }^{\mathrm{b}}$ Centre Algoritmi, University of Minho, 4800-058 Guimarães, Portugal \\ ${ }^{\mathrm{c}}$ Institute for Polymers and Composites IPC/I3N, University of Minho, 4800-058 Guimarães, Portugal \\ ${ }^{\mathrm{d}}$ School of Technology, Polytechnic Institute of Cávado and Ave, 4750-810 Barcelos, Portugal \\ e Life and Health Sciences Research Institute (ICVS), ICVS/3B's - PT Government Associate Laboratory, University of Minho, \\ Campus de Gualtar, 4710-057 Braga, Portugal
}

\section{A R T I C L E I N F O}

\section{Article history:}

Received 15 September 2011

Received in revised form 22 April 2012

Accepted 23 April 2012

Available online 28 April 2012

\section{Keywords:}

Remote patient monitoring

Wireless sensor network

ZigBee

IEEE 802.15.4

\begin{abstract}
A B S T R A C T
This paper presents experimental results of the communication performance evaluation of a prototype ZigBee-based patient monitoring system commissioned in an in-patient floor of a Portuguese hospital (HPG - Hospital Privado de Guimarães). Besides, it revisits relevant problems that affect the performance of nonbeacon-enabled ZigBee networks. Initially, the presence of hidden-nodes and the impact of sensor node mobility are discussed. It was observed, for instance, that the message delivery ratio in a star network consisting of six wireless electrocardiogram sensor devices may decrease from $100 \%$ when no hidden-nodes are present to $83.96 \%$ when half of the sensor devices are unable to detect the transmissions made by the other half. An additional aspect which affects the communication reliability is a deadlock condition that can occur if routers are unable to process incoming packets during the backoff part of the CSMA-CA mechanism. A simple approach to increase the message delivery ratio in this case is proposed and its effectiveness is verified. The discussion and results presented in this paper aim to contribute to the design of efficient networks, and are valid to other scenarios and environments rather than hospitals.
\end{abstract}

(c) 2012 Elsevier B.V. All rights reserved.

\section{Introduction}

Wireless sensor networks (WSNs) have emerged as an enabling technology to interconnect several low-cost, energy-constrained sensor nodes [1-3]. Application areas include environmental monitoring [4-6], precision agriculture $[7,8]$, positioning $[9,10]$ and health monitoring [11-14]. These networks share several similarities with ad hoc networks. However, contrary to ad hoc networks, WSNs are usually comprised of a large number of sensor nodes, which are limited in energy, computational capacity, and memory $[3,15]$. Consequently, the successful implementa-

\footnotetext{
* Corresponding author at: Department of Industrial Electronics, University of Minho, 4800-058 Guimarães, Portugal.

E-mail addresses: hlopez@dei.uminho.pt (H. Fernández-López), jose. afonso@dei.uminho.pt (J.A. Afonso), higino.correia@dei.uminho.pt (J.H. Correia), rsimoes@dep.uminho.pt (R. Simoes).
}

tion of WSNs obliged the development of specific communication protocols.

The first version of the IEEE 802.15.4 protocol [16] was released in 2003 and was largely adopted by the WSN community [17]. It also served as the foundation for other communication protocols, such as ZigBee [18]. The ZigBee protocol has been successfully employed on several sensing applications in spite of the lack of quality-of-service (QoS) guarantees. Since it is based on carrier sense medium access (CSMA), it is susceptible to collisions even in lowtraffic scenarios [19]. Additionally, CSMA-based protocols are susceptible to the hidden-node problem, where some traffic being sent to a receiver may not be detected by another transmitter [6]. In extremely low-load scenarios, the message delivery ratio is not negatively affected by occasional collisions because collided packets are retransmitted if devices are allowed to use acknowledged transmissions. However, as the amount of traffic increases, 
collisions occur more frequently and, consequently, the delivery ratio tends to decrease [20].

Mobility is another aspect that should be considered when specifying a wireless network. The ZigBee protocol support mobility through procedures that reduce the data flow interruption period [18]. However, depending on the application, the data flow interruption periods may be too long resulting in loss of data.

Protocol stack implementation options may negatively influence the performance of networks. For instance, whenever a router receives a message to be relayed, it uses the CSMA-CA mechanism to transmit it to the next hop. However, this mechanism involves a random backoff period during which an incoming message may be processed or ignored, depending on the stack implementation. If an incoming message is repeatedly ignored, it might get lost. A simple strategy to reduce the percentage of message losses in this case is proposed and its effectiveness is verified from simulation.

This paper revisits problems that affect the reliability of nonbeacon-enabled ZigBee networks, which is a key requirement for networks used in data loss intolerant applications. The results presented were determined with the help of simulations and experimental tests. Part of the experimental tests were done while commissioning HM4All (an acronym for Health Monitoring for All), a ZigBee-based physiological monitoring system, in an inpatient floor of Hospital Privado de Guimarães, a Portuguese hospital.

\section{The CSMA-CA algorithm}

In nonbeacon-enabled ZigBee networks, the coordinator is permanently active and, when a device wishes to transfer data, it uses the unslotted version of the CSMA-CA algorithm. Unslotted CSMA-CA constants and attributes are summarized in Table 1 [16], whereas the algorithm is shown in Fig. 1 [16].

Initially, the number of transmission attempts for the current packet (NB) is set to zero and the backoff exponent (BE) takes the value macMinBE. After that, the device waits for a random backoff interval defined in the range from 0 to $\left(2^{B E}-1\right)$ unit backoff periods, where one unit backoff period is equal to 20 symbols $(0.32 \mathrm{~ms}$, in case of the $2450 \mathrm{MHz}$ band). Next, if the clear channel assessment (CCA) function indicates that the channel is idle, the device starts its transmission immediately after its transceiver changes from receiver to transmit mode. On the other hand, if the channel is busy, the device defers its transmission, increments the number of transmission attempts for the current packet, $\mathrm{NB}$, and also increments the $\mathrm{BE}$ value if $\mathrm{BE}$ has not exceeded $a M a x B E$. Then, if the maximum number of transmission attempts has not been exceeded,

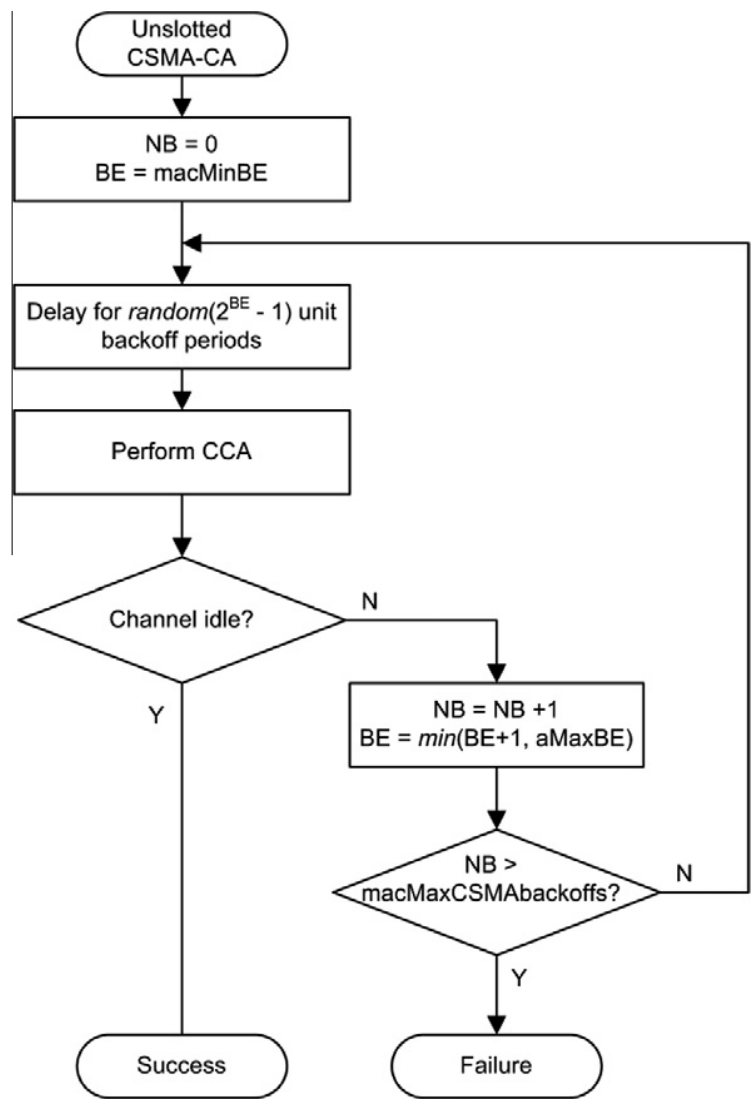

Fig. 1. Unslotted version of the CSMA-CA mechanism [16].

it calculates a new random backoff interval it must wait before assessing the channel again. The device can try to access the channel a maximum number of times defined by the macMaxCSMAbackoffs attribute. When this limit is reached, the MAC sub-layer discards the data and declares a channel access failure.

\section{HM4All architecture}

HM4All is a physiological monitoring system developed to monitor both in and out-patients. Its high-level system architecture is shown in Fig. 2. Data generated by wearable ZigBee-based sensor devices are transported by routers and coordinators to ZigBee-to-IP gateways. Then, data are stored in the data server and made available to monitoring centers and other applications running on wireless portable devices carried by nurses and doctors. In the present implementation, ZigBee networks operate on nonbeaconenabled mode and sensor devices are, at most, two hops away from the network coordinator.

Table 1

Unslotted CSMA-CA constants and attributes for the IEEE 802.15.4-2003 protocol [16].

\begin{tabular}{lll}
\hline MAC constant/attribute & Description & Value \\
\hline macMinBE & The minimum value of the backoff exponent & [0-3], default $=3$ \\
$\begin{array}{l}\text { aUnitBackoffPeriod } \\
\text { aMaxBE }\end{array}$ & The length of the backoff period in symbol periods (SP) \\
macMaxCSMAbackoffs & The maximum value of the backoff exponent & 5 \\
{$[0-5]$, default $=4$} & The maximum number of backoff periods & \\
\hline
\end{tabular}




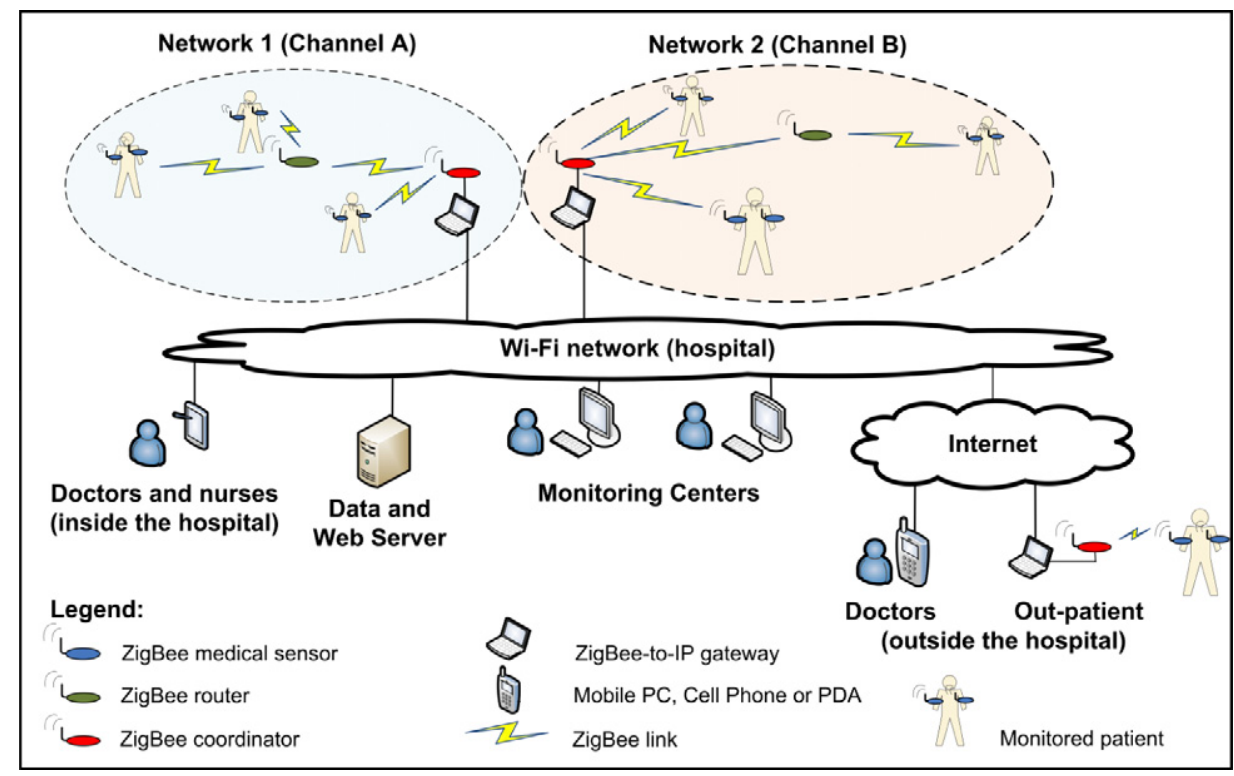

Fig. 2. HM4All architecture.

Two types of physiological sensor devices were developed: (a) a single-channel 3-lead electrocardiogram (ECG) and heart rate (HR) monitor and (b) an axillary thermometer. The amount of physiological data generated by each wireless sensor device is shown in Table 2. Although the ECG and HR are measured by the same device and data is aggregated, the amount of data generated by each function is shown separately. Most of the traffic on the ZigBee network is uplink, form sensor devices to the network coordinator.

\section{Performance of ZigBee-based networks}

This section revisits issues that can impact the performance of ZigBee-based networks, and include simulations or experimental test results. All simulation models were developed using the OMNeT++ discrete event simulator [21]. The experimental settings used have similar characteristics to the motivating application. All devices employed in experiments were based on the JN5139-M00 wireless module, from Jennic [22]. Besides, only nonbeacon-enabled networks based on the ZigBee version 1.0 and default CSMA-CA attributes were used.

\subsection{Mobility}

In case an end device moves away from its parent, the communication flow between them is interrupted. Two scenarios are possible, as shown in Fig. 3: (a) intra-network end device mobility and (b) inter-network end device mobility [23]. Scenarios involving router mobility are studied in [24,25], whereas sink mobility is analyzed in [26]. These scenarios are not discussed here because the motivating application employs sensor devices configured as end devices and does not encompass mobile coordinators or routers.

In case of intra-network end device mobility, the end device moves away from its parent, but it is possible to find another parent among the devices that comprise the network. In case of inter-network mobility, the end device moves away from the coverage area provided by the network and finds another parent in another network. The first case is only possible if the end device operates in a multi-hop topology.

If acknowledged messages are used, an end device may conclude it should search for another parent once the Medium Access Control (MAC) layer reports the occurrence of one or more consecutive failed transmissions to the Application (APP) layer [23]. Another strategy includes monitoring the link quality indication (LQI) values attached to acknowledgement frames as an indicator of channel impairment. If the LQI value falls below a threshold, the end device may search for another parent. Experimental tests were conducted to determine the period of time required by an end device to find and reassociate to a new parent. The time periods presented may serve as reference values for implementers.

Table 2

Amount of physiological data generated by wireless sensor devices.

\begin{tabular}{lll}
\hline Sensor device & $\begin{array}{l}\text { Sampling rate and/or period } \\
\text { between data messages }\end{array}$ & Physiological data generated \\
\hline ECG (modified Lead I) & $200 \mathrm{~Hz} / 500 \mathrm{~ms}$ & $1200 \mathrm{bps}(2: 1 \mathrm{compressed} \mathrm{data)}$ \\
HR & $3 \mathrm{~s}$ & 1 byte every $3 \mathrm{~s}$ \\
Axillary temperature & $1 \mathrm{~min}$ & 1 byte every min \\
\hline
\end{tabular}




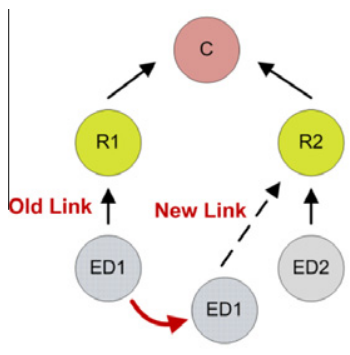

(a)

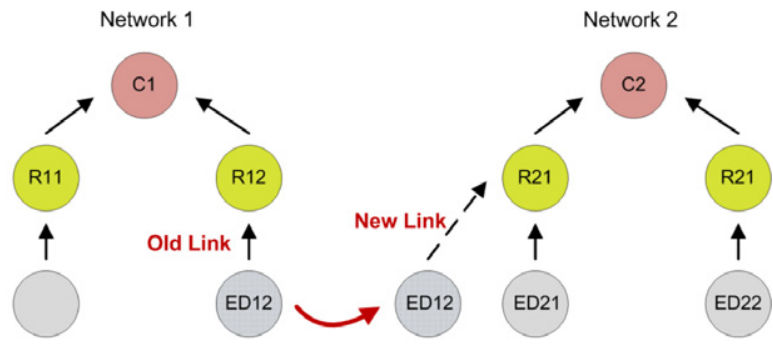

(b)

Fig. 3. End device mobility: (a) intra-network and (b) inter-network.

In order to find a new parent within the same network, as illustrated in the scenario shown in Fig. 3a, the end device should perform a MAC layer association procedure specifying only the channel it is presently operating [16]. The mean rejoining time measured using the SNA protocol analyzer [27], in a quiet environment, was equal to $762 \mathrm{~ms}$.

In case the orphaned end device is unable to find another parent to associate with, it may opt to search for another network. This scenario is illustrated in Fig. 3b. Initially, the end device scans all channels or a subset of channels and, if another network is found, it tries to associate. Two ZigBee networks (one operating in channel 25 and the other in channel 26) were used to verify the time required by an end device to move from one parent, in a given network, to another one in another network. The end device was programmed to start measuring time just before scanning all ZigBee channels in search of networks and to stop measuring time just after the successful conclusion of the association process. The process was repeated several times and the required time to complete the reassociation procedure lasted between $1912 \mathrm{~ms}$ and $1919 \mathrm{~ms}$

In both cases, the data flow is interrupted during reassociation. Besides, whereas the data flow from the end device to the network is resumed after the successfully completion of the reassociation process, the data flow in the opposite direction can only be resumed when a new route to the device is established. In the case of HM4All, during the reassociation process, high data-rate transfer sensors, like ECG sensor devices, would lose one or more messages. Conversely, a heart rate or temperature sensor device, which presents lower data transfer rate, may experience no data loss. Short data flow interruption periods might be tolerated in several settings involving non-acute patients, which are the scenarios envisioned for health monitoring systems based on the ZigBee protocol.

\subsection{Router deadlock}

In multi-hop topologies, apart from end devices, routers also compete to access the wireless channel, which aggravates the contention. Fig. 4 shows a temporal sequence of frames exchanged by ZigBee-based nodes in a nonbeacon-enabled 2-hop tree network comprised of two end devices, ED1 and ED2, associated to a router which is one hop away from the network coordinator (COORD). ED1 transmits a data frame just after ED2 receives an acknowledgement frame from the router. This particular router can neither receive the data frame transmitted by ED1 because it has already initiated the CSMA-CA channel access mechanism, nor relay the received data frame from ED2 because it senses the channel busy. ED1 extinguishes all possible retries and, consequently, its message is lost. Finally, as shown in Fig. 4, the router relays the frame transmitted by ED2. However, in other occasions, the router can drop this data frame if it reaches the maximum number of channel access retries.

The packet timeline shown in Fig. 5 exemplifies the contention problem just described. The packets were captured using the 2400E sensor network adapter [28] and the SNA protocol analyzer [27] from a 2-hop ZigBee-based tree network that included four end devices (network addresses 0x1430 - 0x1433) associated to a router (network address 0x0001). At intervals of approximately $500 \mathrm{~ms}$, each end device generated one message that was addressed to the network coordinator (network address 0x0000). The first packet shown is packet 1 . This packet was sent by end device $0 \times 1431$ and acknowledged by the router (in packet timeline shown, acknowledgment frames are only displayed in the Channel Summary line). The acknowledgment frame is followed by a failed transmission (packet 2) and two successive retries made by the end device 0x1433. Then, the router finds the channel idle and relays packet 1. A few milliseconds after the acknowledgement frame sent by the coordinator to the router, the end device 0x1433 makes a successful retry and receives an acknowledgement frame transmitted by the router. In this case, despite having made three retries after a failed transmission, the end device 0x1433 was able to deliver its message.

Packet 3 was also sent by the end device $0 \times 1431$ to the router. The acknowledgment frame sent by the router is followed by a failed transmission (packet 4 ) and three retries (the maximum number of retries allowed by the protocol) made by the end device 0x1433. After these packets, the router successfully relays packet 3 . In this case, the end device 0x1433 failed to deliver its message.

The message loss and the retries would have been avoided if the router could receive incoming packets during the backoff period. However, that is not possible for Jennic's protocol implementation supplied with JN5139 modules [22]. Such behavior is not an infringement of the IEEE 802.15.4 standard because it does not specify 


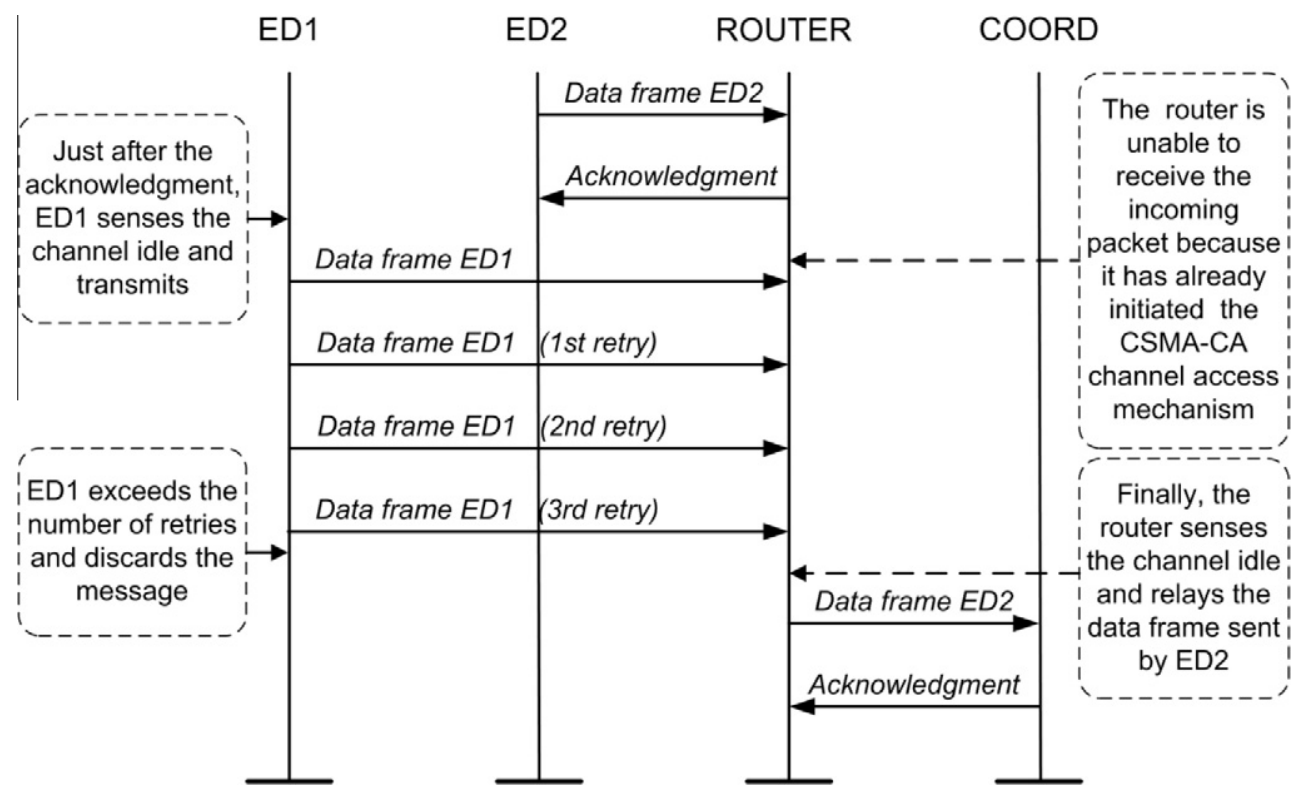

Fig. 4. Router deadlock example.

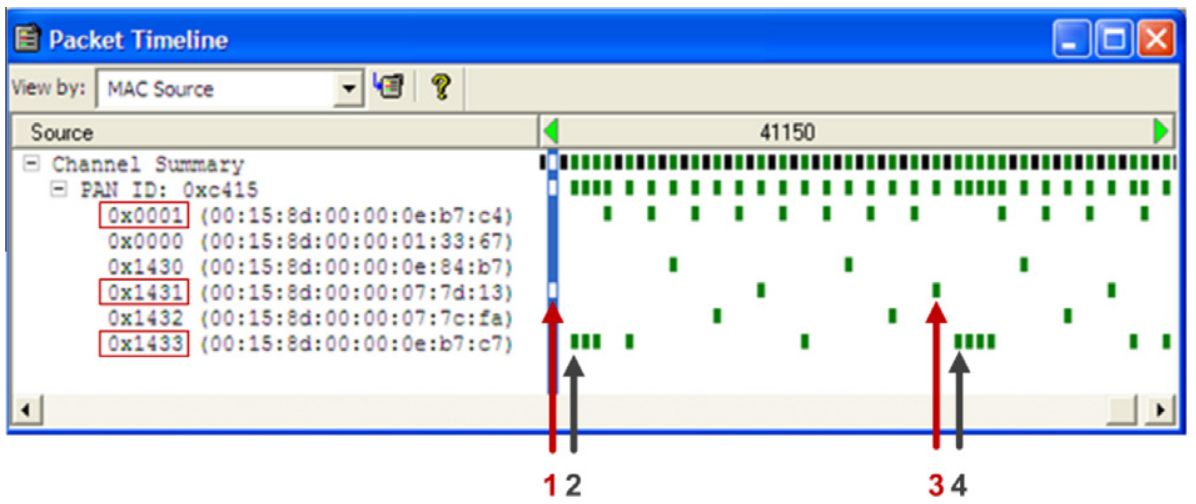

Fig. 5. Packet timeline captured while testing a 2-hop ZigBee-based tree network.

what procedure must be followed during the backoff part of the CSMA-CA mechanism (described in Section 7.5.1.3 of [16]). Therefore, other manufacturers might have opted for ignoring incoming packets for the duration of backoff.

Considering that most commercial platforms do not allow developers to change the stack implementation, if the CSMA-CA mechanism ignores incoming packets during backoff periods, it is possible that router deadlocks occur. In this case, a simple strategy can be used to reduce the number of message losses. If the router had more priority to access the channel than the end devices, the contention problem described would have occurred less frequently. This can be achieved by setting different values of the MAC layer attribute macMinBE for each type of device. In order to confirm it, we have estimated, from simulation, the delivery ratio values considering a 2-hop tree network consisting of a variable number of end devices associated to a single router, as shown in Fig. 6 .

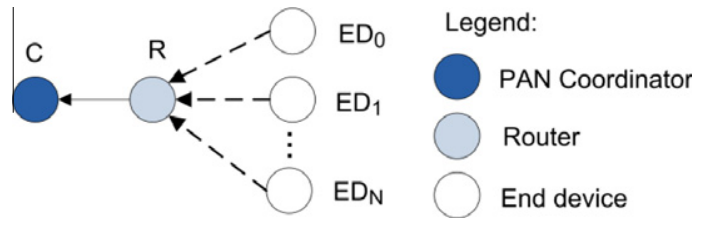

Fig. 6. A 2-hop tree network.

Different values of the time interval between messages generated by end devices $(0,1$ or $2 \mathrm{~ms})$ were used in each simulation set. These small values were chosen to cause contention between end devices. In addition, two different values of the router's MAC attribute $\operatorname{macMinBE}(3$ or 0 ) and a crescent number of end devices were considered. Each end device generated one ECG data message consisting of 114 bytes at fixed intervals of $500 \mathrm{~ms}$. Correctly received messages were acknowledged. All MAC attributes, except the router's macMinBE attribute, assumed default values. 
Each simulation run ended when the coordinator correctly received 100,000 messages.

Table 3 contains the results obtained, where each delta time column specifies the time difference between successive messages generated by all end devices. For instance, if delta time is equal to 0 , all end devices generate a packet every $t(\mathrm{~ms})=n * 500$, where $n$ is the simulation cycle number. Alternatively, if delta time is equal to $1 \mathrm{~ms}$ and the number of end devices is equal to 2, one end device generates a packet every $t(\mathrm{~ms})=n * 500$, whereas the other generates a packet every $t(\mathrm{~ms})=(n * 500)+1$. Each macMinBE column ( $\operatorname{macMinBE}=0$ and macMinBE $=3$ ) shows the value of the resulting delivery ratio. Every delta $D R$ column shows the relative improvement in the delivery ratio value when the router's macMinBE attribute is altered from 3 to 0 .

As shown in Table 3, the delivery ratio values increase significantly when the router's macMinBE attribute is set to 0 . For instance, if two end devices generate messages at the same time and the router's attribute macMinBE is set to 3 , the resulting delivery ratio value is equal to 0.684 . However, if the router's macMinBE attribute is set to 0 , the delivery ratio value increases to 0.828 , an improvement of $21 \%$. On the other hand, if the interval between messages generated by two devices is very small, this strategy alone is not enough to prevent packets from being lost.

Section 5.2 presents experimental results involving nonbeacon-enabled networks where, occasionally, and during a certain period of time, a pair of sensor nodes loses packets mainly due to router deadlocks.

\subsection{Clock drift in nonbeacon-enabled networks}

The clock drift is often expressed in parts per million (ppm) and gives the number of additional or missing oscillations a clock makes in the amount of time needed for one million oscillations at the nominal rate. Apart from manufacturing inaccuracies, the frequency of oscillators may drift because of aging effects and temperature variations, among other causes [29]. Since sensor nodes run on relatively cheap oscillators, large clock drifts are expected. If no synchronization method is applied to a WSN, nodes keep no time relationship between their clocks.

In a nonbeacon-enabled WSN, the time differences between transmissions from different sensor nodes can vary considerably in a relatively small amount of time due to clock drift. The following experience was run to observe the evaluation of the relative time differences between transmissions done by sensor nodes. Four test boards based on JN5139 modules were programmed to generate one message at $500 \mathrm{~ms}$ intervals. These modules have a maximum clock drift of $40 \mathrm{ppm}$ (that is, their clocks can deviate up to $40 \mu \mathrm{s}$ in $1 \mathrm{~s}$ ) [22]. A nonbeacon-enabled star network was used and packets were captured using the SNA network analyzer [27]. Sensor nodes were activated and, periodically, the transmission times of successive messages from all sensors were recorded. Collision avoidance was disabled during the first iteration of the CSMACA algorithm (that is, the MAC layer macMinBE attribute was set to 0 ) to avoid end device backing off before the first attempt to assess the channel.

Fig. 7 shows time measurements executed at 10:00 am and at 10:30 am. The squares represent the sensor nodes, ED1 to ED4, and the numbers on top of the nodes represent the time, in milliseconds, when each sensor node transmitted a message. The first timeline shows that ED3 and ED4 transmitted a message at 10:00:00.647 (time format: hh:mm:ss.ms) and at 10:00:00.654, respectively. Thus, ED3 transmitted a message $7 \mathrm{~ms}$ before ED4 transmitted its message. For a few seconds, it was observed that this order was maintained. However, in a second measurement done half-hour later, it was verified that the order of transmission of messages had changed due to the difference in the clock drifts of sensor nodes. ED4 transmitted a packet $11 \mathrm{~ms}$ before ED3, and this difference was maintained, at least, for a few seconds. During this half-hour interval, ED3 and ED4 have contended for the channel and it is possible that some of their packets have collided.

\subsection{Hidden-nodes}

A generic hidden-node scenario is illustrated in Fig. 8a, where the transmission range of nodes $\mathrm{A}$ and $\mathrm{B}$ are represented by circles drawn around the nodes. If $A$ is transmitting to $C$, and B starts to transmit to D (B's carrier sense fails), then A's and B's packets collide at C. In this case, the transmission from $A$, which is hidden from $B$, is corrupted by B's transmission. A more severe problem is illustrated in Fig. 8b. This situation consists on A and B transmitting towards a common receiver, $C$. In this case, if B starts to transmit while $\mathrm{A}$ is transmitting (or vice versa), both packets are corrupted.

A simulation analysis was performed to estimate the negative effect of hidden-nodes in a nonbeacon-enabled ZigBee-based star network. Fifty simulation runs were exe-

Table 3

Delivery ratio results obtained from simulation. Delta time is expressed in milliseconds (ms).

\begin{tabular}{|c|c|c|c|c|c|c|c|c|c|}
\hline \multirow[t]{3}{*}{ Number of end devices } & \multicolumn{3}{|c|}{ Delta time $=0$} & \multicolumn{3}{|c|}{ Delta time $=1 \mathrm{~ms}$} & \multicolumn{3}{|c|}{ Delta time $=2 \mathrm{~ms}$} \\
\hline & \multicolumn{2}{|c|}{ macMinBE } & \multirow[t]{2}{*}{ Delta DR (\%) } & \multicolumn{2}{|c|}{ macMinBE } & \multirow[t]{2}{*}{ Delta DR (\%) } & \multicolumn{2}{|c|}{ macMinBE } & \multirow[t]{2}{*}{ Delta DR (\% } \\
\hline & 3 & 0 & & 3 & 0 & & 3 & 0 & \\
\hline 2 & 0.684 & 0.828 & 21 & 0.745 & 0.902 & 21 & 0.791 & 0.963 & 22 \\
\hline 3 & 0.544 & 0.710 & 31 & 0.597 & 0.807 & 35 & 0.572 & 0.797 & 39 \\
\hline 4 & 0.437 & 0.598 & 37 & 0.465 & 0.691 & 49 & 0.461 & 0.671 & 46 \\
\hline 5 & 0.352 & 0.503 & 43 & 0.362 & 0.570 & 58 & 0.376 & 0.589 & 57 \\
\hline 6 & 0.290 & 0.430 & 48 & 0.295 & 0.492 & 67 & 0.322 & 0.529 & 64 \\
\hline
\end{tabular}




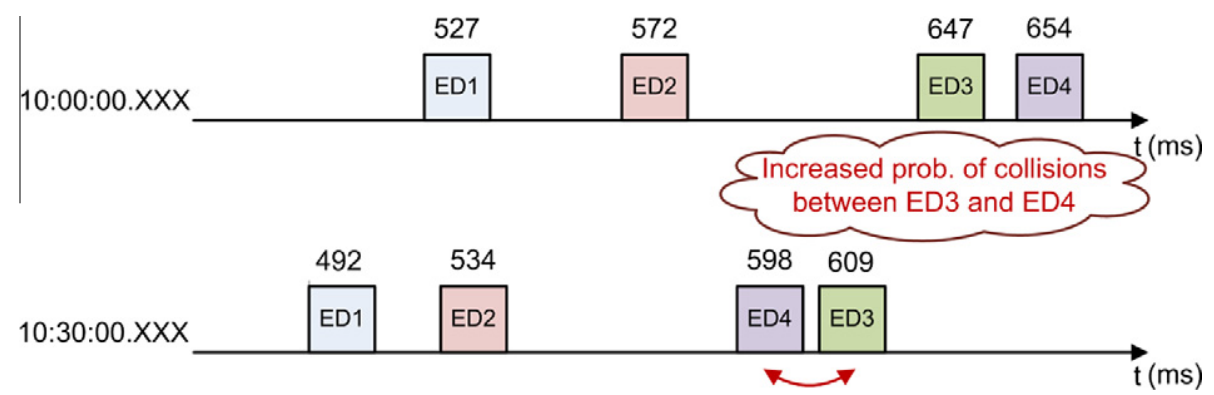

Fig. 7. Clock drift measurements in a nonbeacon-enabled ZigBee-based star network.

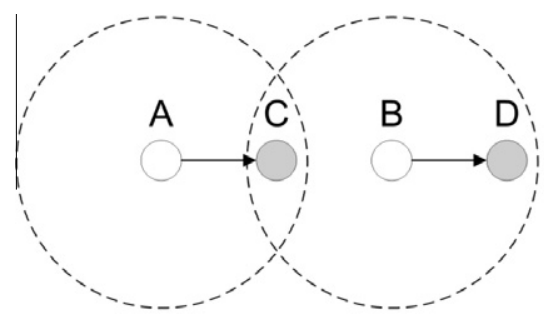

(a)

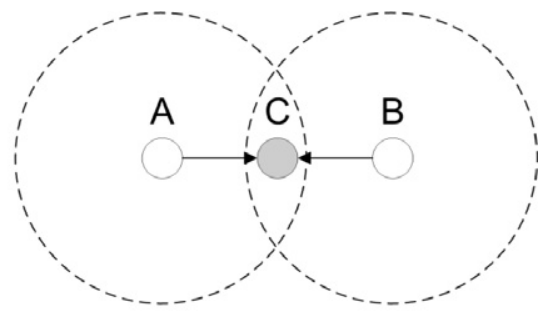

(b)

Fig. 8. Hidden-node scenarios: (a) different receivers and (b) same receiver.

cuted, each one considering a number of nodes ranging from 1 to 50. Each node generated a data packet consisting of 114 bytes every $250 \mathrm{~ms}$. Only acknowledged transmissions were considered. Default values of the CSMA-CA attributes were used. Each simulation run ended when the number of messages correctly received by the network coordinator reached 100,000 .

Fig. 9 presents the variation in the delivery ratio as a function of the number of nodes for a crescent percentage of hidden-nodes from 0 to $20 \%$. If no hidden-nodes are present, high delivery ratio values are achieved with a relatively high number of nodes. However, even a small percentage of hidden-nodes can cause a considerable increase in the number of collisions and, consequently,

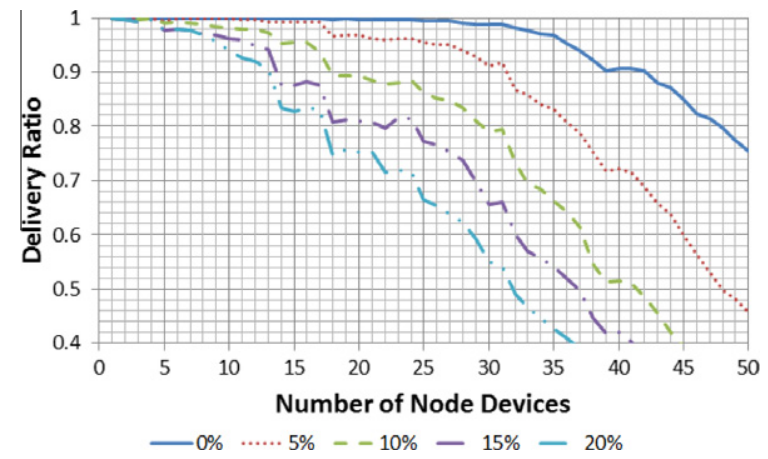

Fig. 9. Delivery ratio curves for a crescent number of nodes and different percentages of hidden-nodes. These results were obtained from simulation for a star network seriously affects the performance of the network. For instance, if no hidden-nodes are present more than $99.0 \%$ of the messages generated by up to 27 nodes are correctly delivered. However, if the percentage of hidden-nodes is increased to $5 \%$ or $10 \%$, the number of ECG nodes must be reduced to 18 or 8 , respectively, to achieve the same delivery ratio.

Section 5.3 presents experimental results from star networks comprised of ECG sensor devices that operated in the absence and in the presence of a high percentage of hidden-nodes.

\section{Communication performance evaluation of HM4All}

\subsection{Experimental setting for delivery ratio tests}

The floor plan of the inpatient area where the system was commissioned is shown in Fig. 10. This floor contains 12 patient rooms, R201-R209 and R211-R213, areas accessible to patients and restricted areas. The small yellow numbered squares refer to elevated spots (approximately $2.2 \mathrm{~m}$ from the ground level) with energy plugs available. Blue rectangles refer to $\mathrm{Wi}-\mathrm{Fi}$ access points (APs).

In this paper, all results presented involve nonbeaconenabled ZigBee-based networks. The setting presented in Fig. 11a was used to perform tests using a star network. The coordinator was positioned on the hallway, near the entrance of the consultation room (CONS. ROOM), whereas end devices (represented in grey) were placed at rooms R201-R204. Fig. 11b presents the settings used to test a 2-hop tree network. Two routers were added and the coordinator was brought into the consultation room (the 


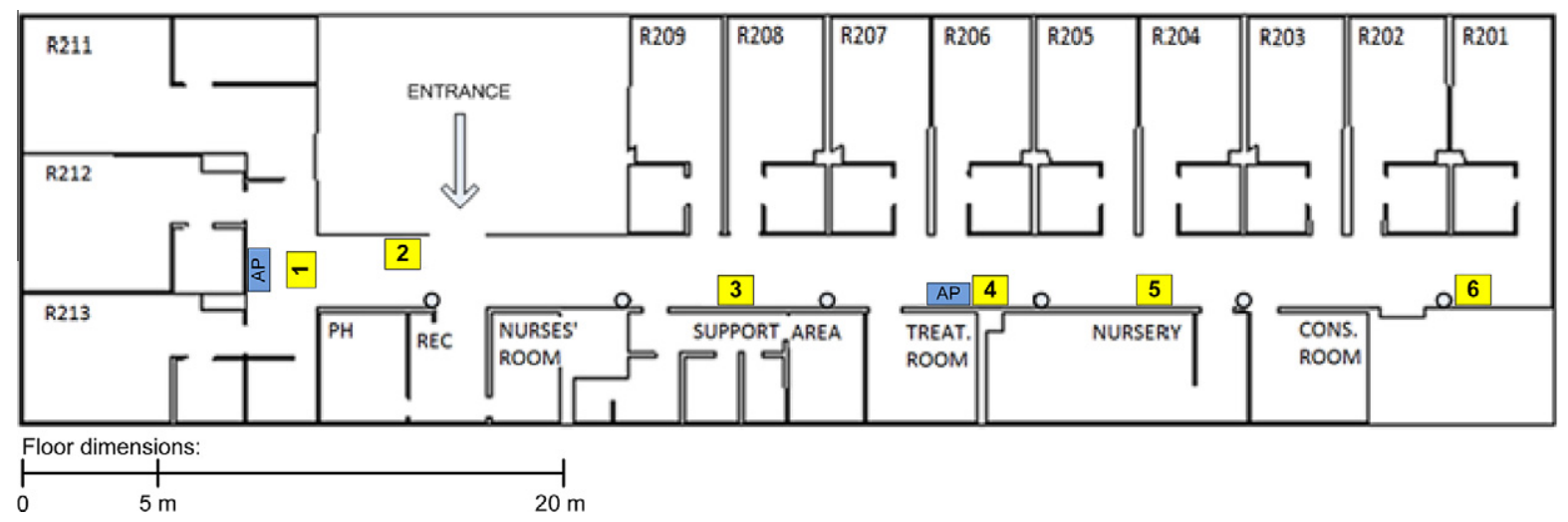

Fig. 10. Floor plan of the in-patient floor where HM4All was commissioned.

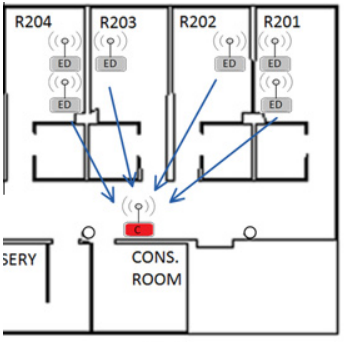

(a)

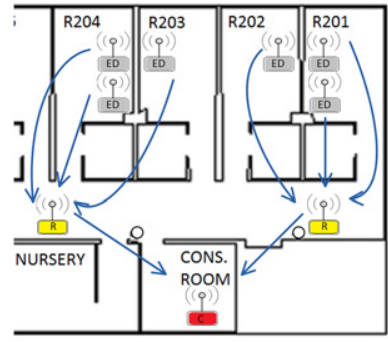

(b)

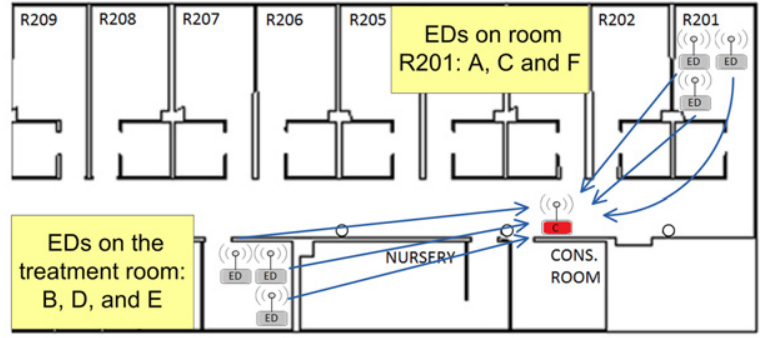

(c)

Fig. 11. Test settings: (a) star topology, no hidden-nodes; (b) 2-hop tree topology, no hidden-nodes; and (c) star topology, 50\% of hidden-nodes.

routers and the coordinator are represented, respectively, in yellow and red ${ }^{1}$ ). According to previous measurements, no hidden-nodes were present on these settings.

The setting shown in Fig. 11c was used to evaluate the performance of a star network in the presence of hiddennodes. The coordinator was placed just outside the consultation room, whereas room R201 and the treatment room each received three end devices. As previously verified, end devices placed at one room could not communicate or detect the transmissions attempts made by end devices placed at the other room. A test application was developed to monitor the messages received by the coordinator. Additionally, all packets exchanged during the tests were recorded using the SNA network analyzer and a 2400E network adapter.

During the tests, end devices were positioned near the head of patients' beds. Six end devices (A-F) were used and each one generated the data traffic of ECG sensor devices, which consists of one 114-byte data message every $500 \mathrm{~ms}$. All successfully received messages were acknowledged at each hop. No other traffic was generated. Default values of MAC layer attributes were used. All tests were executed using ZigBee channel 26 to avoid wireless local area network (WLAN) interference. No restriction was imposed regarding the use of any wireless equipment. All tests were supervised by the hospital staff.

\footnotetext{
${ }^{1}$ For interpretation of color in Figs. 2, 3, 5-7, and 9-14, the reader is referred to the web version of this article.
}

For all field tests, the mean delivery ratio and the delivery ratios achieved by individual end devices were sequentially calculated at 2-s intervals using a 10 -s running window.

\subsection{Delivery ratio analysis}

Table 4 presents the mean delivery ratio obtained for the tests using the star and the 2-hop tree topologies shown in Fig. 11a and b. As shown, in the absence of hidden-nodes, the star network was able to deliver almost all messages generated (only 7 out of 175,738 generated messages were lost); whereas the 2-hop tree network achieved a delivery ratio of $98.56 \%$.

Fig. 12 shows the first half of the results computed for the 2-hop tree network shown in Fig. 11b. The second half of the results is similar. The $x$-axis represents a temporal sequence of 2-s time intervals, whereas the $y$-axis represents the delivery ratio measured using the 10-s running window. Each curve shows the delivery ratio values measured for each end device. As shown, during the first minutes of test, no packet is lost because the sensor devices do

Table 4

Mean delivery ratio values for ZigBee-based nonbeacon-enabled networks consisting of six ECG sensors. No hidden-nodes were present.

\begin{tabular}{lcl}
\hline Network topology & Duration $(\mathrm{h})$ & Mean DR $(\%)$ \\
\hline Star network & 5.1 & 100 \\
2-hop, tree network & 16.7 & 98.56 \\
\hline
\end{tabular}




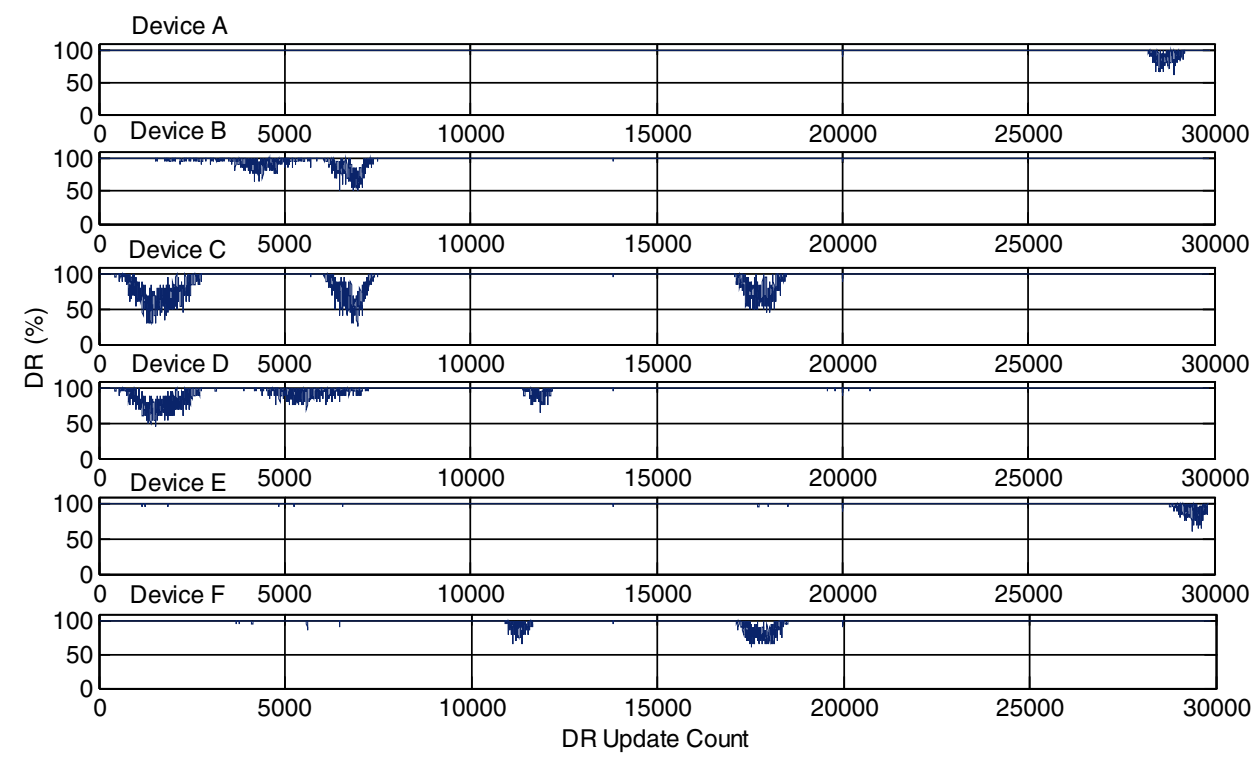

Fig. 12. Delivery ratio per 2 -s interval calculated using a 10 -s (20-message length) window for the first part of the test involving a 2 -hop tree network with no hidden-nodes.

not contend to access the wireless channel. However, after about half an hour, the delivery ratio for end devices $C$ and $D$ decreases for half an hour, approximately, before it starts to increase again. As observed, this situation repeats for other pairs of end devices.

As shown in Fig. 12, message losses periods observed for pairs of end devices are transitory. Although end devices had been programmed to generate a message at fixed $500 \mathrm{~ms}$ intervals, their output rates varied along the time due to differences in their clock drifts. Therefore, sporadically, time differences between messages generated by two end devices were sufficiently small to generate contention, which resulted in message losses. These message losses were mostly likely caused by router deadlock situations, as shown in Section 4.2. An additional amount of messages were lost due to successive collisions between contending devices. However, message losses due to successive collisions are relatively rare because, in case hidden-nodes are not present, these situations are efficiently handled by the MAC layer.

\subsection{Hidden-node analysis}

Table 5 presents the mean delivery ratio obtained for the test using the star network shown in Fig. 11c, where three of the end devices were hidden from the other three. The network was operational for almost $8 \mathrm{~h}$, but data recording was interrupted for, approximately, 50 min. Dur-

Table 5

Mean delivery ratio values for a ZigBee-based star network consisting of six ECG sensors, where three of which were hidden from the other three.

\begin{tabular}{lll}
\hline Part & Duration $(\mathrm{h})$ & Mean DR $(\%)$ \\
\hline 1 & 2.8 & 83.96 \\
2 & 4.2 & 99.96
\end{tabular}

ing the first and second parts of the test, data was recorded for around $2.8 \mathrm{~h}$ and $4.2 \mathrm{~h}$, respectively.

Fig. 13 shows the delivery ratio curves that correspond to the first part of the test. As shown, the curves for end devices $A$ and $E$ have similar shapes, that is, when the delivery ratio for A drops, the same effect is observed for E. Accordingly, when A stops loosing packets, the same occurs to E. These end devices are hidden from each other (A was placed at room R201, whereas E was placed at the treatment room). Consequently, during contention periods, which occur from times to times due to differences in clock drifts, hidden pairs of end devices loose a large percentage of their generated messages due to hidden-node collisions that could not be solved by the CSMA-CA mechanism. The same have occurred to end devices $D$ and $F$ and, just before the end of the test, to devices A and B.

As shown in Fig. 14, during the second part of the test, all end devices achieve a high delivery ratio. The negative results during the first part of the test are not observed because contention between devices did not occur resulting in most of the messages generated by end devices being successfully received by the coordinator.

The test results presented show that nonbeacon-enabled CSMA-based networks cannot maintain a high delivery ratio in presence of hidden-nodes even when submitted to moderate traffic load. ${ }^{2}$ Consequently, in applications that require high communication reliability, it is necessary to avoid the presence of hidden-nodes or to employ a strategy that mitigates their effect.

\footnotetext{
$\overline{2}$ A transaction involving the transmission and acknowledgment of an ECG data message in a star network occupies the channel for $4.512 \mathrm{~ms}$. Accordingly, six transactions (corresponding to six ECG sensors) take $27.072 \mathrm{~ms}$, and correspond to a channel utilization of $5.4 \%$ (all six transactions should be concluded in $500 \mathrm{~ms}$ ).
} 


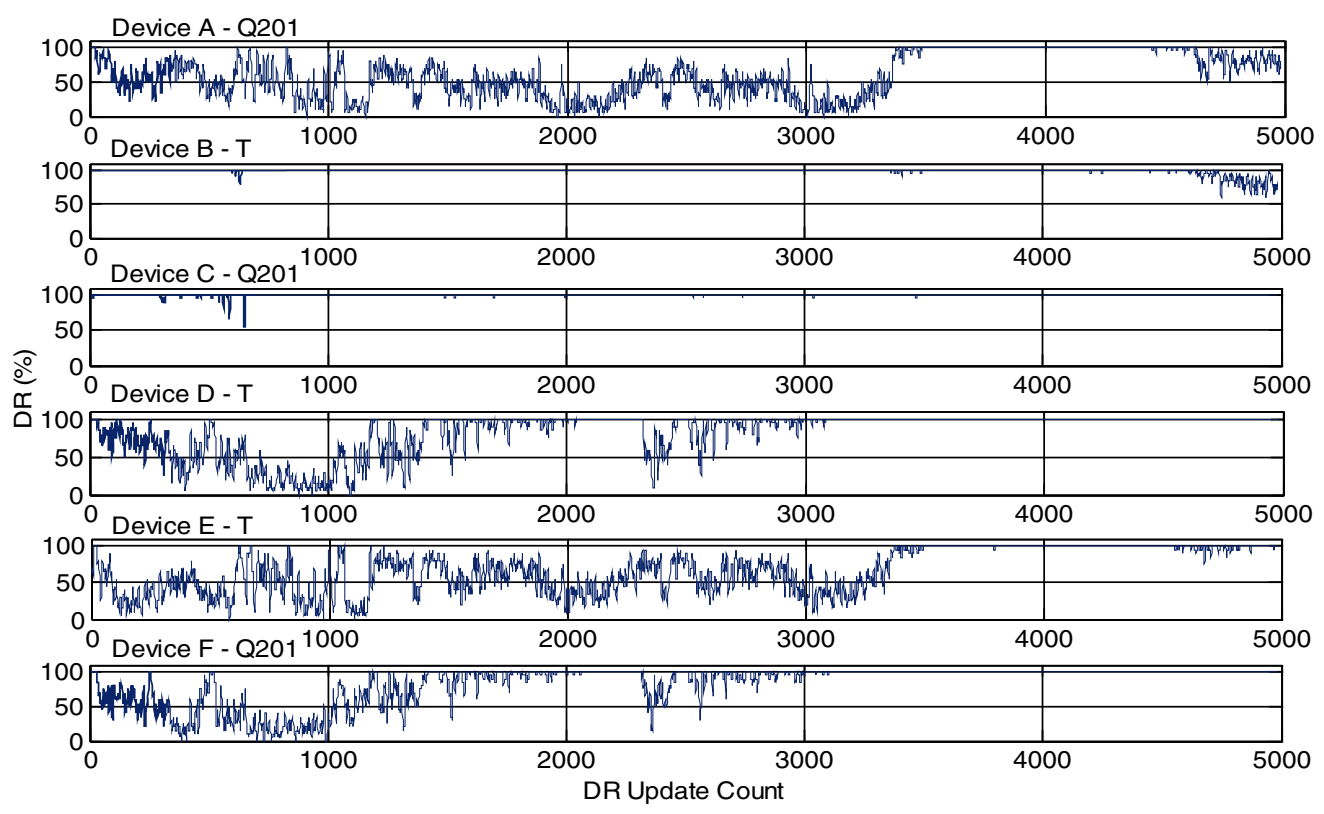

Fig. 13. Delivery ratio per 2-s interval calculated using a 10-s window for the first part of the test involving a star network with 50\% of hidden-nodes.

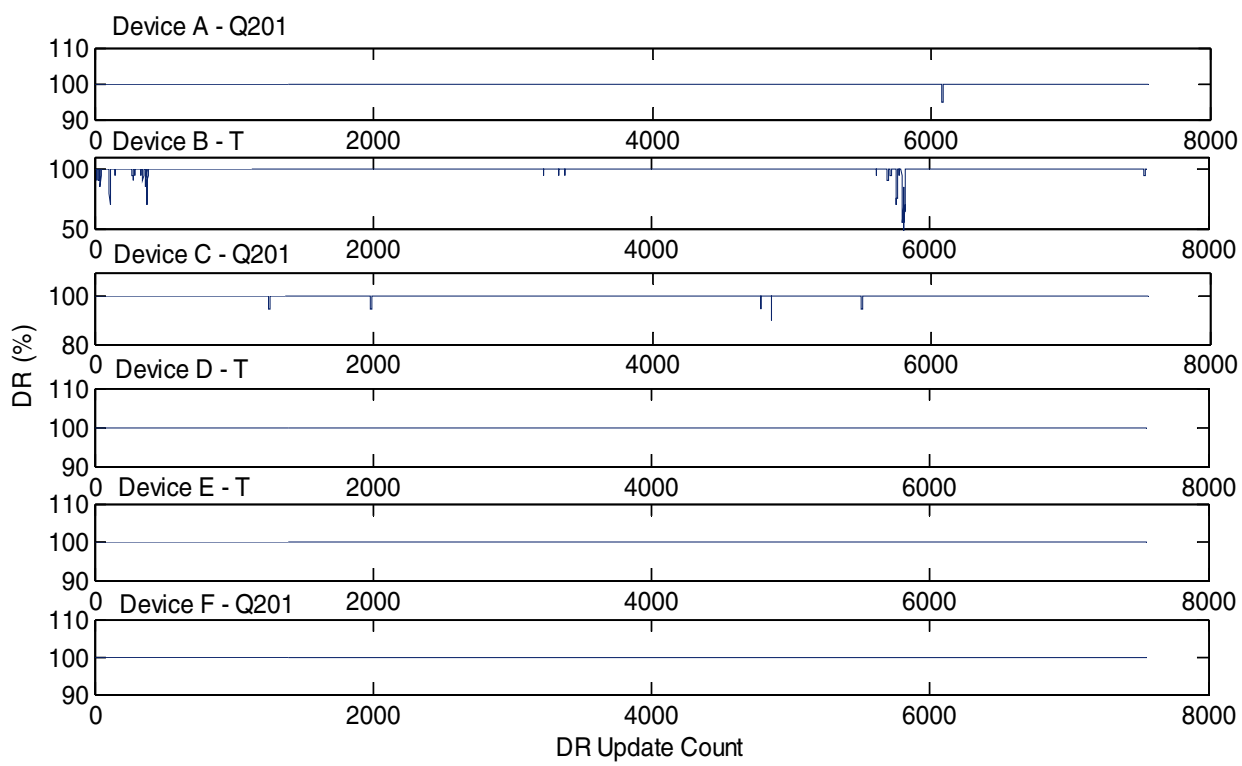

Fig. 14. Delivery ratio per 2 -s interval calculated using a 10 -s window for the second part of the test involving a star network with $50 \%$ of hidden-nodes.

\section{Related work}

The absence of a specific mechanism to avoid the hidden-node problem in ZigBee networks motivated some researchers to consider specific scenarios and propose strategies to alleviate this problem. Di Marco et al. [30] proposed a framework for the analysis of multi-hop networks using the unslotted IEEE 802.15.4 MAC. His work extends the original contribution of Bianchi [31] to include important features of multi-hop networks, as heterogeneous distribution of the traffic and hidden terminal nodes. Koubaa et al. [32], Lain-Jinn et al. [33] and Ruzzelli et al. [34] proposed original mechanisms to mitigate the hidden-node problem in WSNs. However, these mechanisms have considered beacon-enabled networks consisting of static nodes and changes to the protocol specification. This scenario is not considered in this paper because it focuses on nonbeacon-enabled networks and mobile nodes. Moreover, changes introduced in the protocol implementation may cause interoperability issues between sensors from several vendors, what is considered undesirable. 
Mobility, which is referred as portability in the Health Care Profile specification [23], is addressed by the ZigBee specification through the rejoin procedure [18]. Chen, Sun and Liang evaluated the mobility support provided by ZigBee [24]. They concluded that a mobile router experiences less packet losses than a mobile end device because a router can quickly establish a new route in case the old one fails. Additionally, they conclude that mesh routing is more robust than tree routing, although the first one is more demanding in terms of control traffic. In [35], Mu and Liu compared the performance of routing methods (suppress, enable or force route discovery) used by routing-capable ZigBee devices and demonstrated that suppressing route discovery is advantageous in stable networks, whereas enabling route discovery is the best strategy for unstable networks. These options are not explored in this paper because all sensor devices are configured as end devices and, consequently, are not routing capable. In [36], Stevanovic and Vlajic studied the performance of IEEE 802.15.4-based networks with a mobile data sink. In this paper, the data sink, which is also the network coordinator, is fixed.

\section{Conclusion}

In this paper, HM4All, a remote physiological monitoring system based on nonbeacon-enabled ZigBee networks, is described. Besides, relevant problems that affect the communication performance of ZigBee-based nonbeaconenabled networks are revisited. Finally, the results of the communication performance evaluation of HM4All are presented and discussed.

Mobility is supported by the ZigBee protocol. However, in all cases, it implies temporary data flow interruption. Laboratory tests performed using test boards based on the JN5139 communication module, from Jennic, have shown that, after an end device recognizes it has lost communication with its parent, it takes less than $1 \mathrm{~s}$ to reassociate to a new parent, in the same network. In case the end device must search for a new parent in another network, the process that involves nearby networks discovering and new parent association requires, approximately, $2 \mathrm{~s}$. These data flow interruptions may be acceptable to some applications that involve remote monitoring of ambulatory, non-acute patients.

As the IEEE 802.15.4 protocol does not specify if a device should interrupt or not the backoff part of the CSMA-CA mechanism to receive incoming packets, some vendors might have opted for neglecting these packets. Under this condition, which was termed router deadlock, multi-hop networks may experience contention that result in messages being lost. In case it is not possible to modify the MAC implementation, it was proposed to assign zero to the value of the MAC attribute macMinBE of routers while maintaining the default value of 3 to this MAC attribute of end devices to minimize the amount of message losses. Simulations that corroborate with this assumption were also presented.

The negative impact of hidden-nodes in nonbeacon-enabled ZigBee-based networks is evaluated from simulation and experimental tests carried out during the commission of HM4All in an inpatient floor of a Portuguese hospital. Under the absence of hidden-nodes and using six nodes that generated the traffic of ECG sensor devices, the system was able to achieve a delivery ratio of $100 \%$ and $98.56 \%$, respectively, for star and 2-hop tree topologies. As discussed, most of the message losses observed for the 2hop tree topology could have been avoided if the router would have been able to receive incoming messages during the backoff period of the CSMA-CA mechanism. However, under the presence of hidden-nodes, the communication performance of the network degraded considerably during periods of contention.

\section{Acknowledgments}

Authors would like to thank Dr. João Paulo Carmo for the valuable comments and Mr. Pedro Macedo for programming the simulations. They also want to thank Mr. Nélson Brito, Mr. Teófilo Leite and Ms. Teresa Moura, from Hospital Privado de Guimarães, for the encouragement and support. This work has been supported by the Portuguese Foundation for Science and Technology (FCT), Lisbon, through the $3^{\circ}$ Quadro Comunitário de Apoio, the MITPortugal, POCTI and FEDER programs, project PEst-C/ CTM/LA0025/2011, and the FCT grant SFRH/BD/39408/ 2007. Clinical and financial support for the case-study has been provided by Grupo AMI - Assistência Médica Integral (Casa de Saúde Guimarães, SA), Portugal, under the partnership established between this healthcare company and the University of Minho.

\section{References}

[1] R. Verdone, D. Dardari, G. Mazzini, A. Conti, Wireless Sensor and Actuators Networks: Technologies, Analysis and Design, Academic Press, London, UK, 2008.

[2] D. Estrin, L. Girod, G. Pottie, M. Srivastava, Instrumenting the world with wireless sensor networks, in: International Conference on Acoustics, Speech, and Signal Processing, Salt Lake City, 2001, vol 2034, pp. 2033-2036.

[3] M. Tubaishat, S. Madria, Sensor networks: an overview, IEEE Potentials 22 (2003) 20-23.

[4] A.M. Melesse, Q. Weng, P.S. Thenkabail, G.B. Senay, Remote sensing sensors and applications in environmental resources mapping and modelling, Sensors 7 (2007) 3209-3241.

[5] P. Wang, Z. Sun, M.C. Vuran, M.A. Al-Rodhaan, A.M. Al-Dhelaan, I.F. Akyildiz, On network connectivity of wireless sensor networks for sandstorm monitoring, Computer Networks 55 (2011) 1150-1157.

[6] D. Dardari, A. Conti, C. Buratti, R. Verdone, Mathematical evaluation of environmental monitoring estimation error through energyefficient wireless sensor networks, IEEE Transactions on Mobile Computing 6 (2007) 790-802.

[7] L. Ruiz-Garcia, L. Lunadei, P. Barreiro, I. Robla, A review of wireless sensor technologies and applications in agriculture and food industry: state of the art and current trends, Sensors 9 (2009) 4728-4750.

[8] J. Hwang, H. Yoe, Study on the context-aware middleware for ubiquitous greenhouses using wireless sensor networks, Sensors 11 (2011) 4539-4561.

[9] D. Dardari, A. Conti, U. Ferner, A. Giorgetti, M.Z. Win, Ranging with ultrawide bandwidth signals in multipath environments, Proceedings of the IEEE 97 (2009) 404-426.

[10] M.Z. Win, A. Conti, S. Mazuelas, S. Yuan, W.M. Gifford, D. Dardari, M. Chiani, Network localization and navigation via cooperation, IEEE Communications Magazine 49 (2011) 56-62.

[11] A. Hande, T. Polk, W. Walker, D. Bhatia, Self-powered wireless sensor networks for remote patient monitoring in hospitals, Sensors 6 (2006) 1102-1117 
[12] H. Fernandez-Lopez, J.A. Afonso, J.H. Correia, R. Simões, HM4All: A vital signs monitoring system based in spatially distributed ZigBee networks, in: Fourth International Conference on Pervasive Computing Technologies for Healthcare, Munich, 2010, pp. 1-42010.

[13] K. Lorincz, D.J. Malan, T.R.F. Fulford-Jones, A. Nawoj, A. Clavel, V. Shnayder, G. Mainland, M. Welsh, S. Moulton, Sensor networks for emergency response: challenges and opportunities, IEEE Pervasive Computing 3 (2004) 16-23.

[14] H. Alemdar, C. Ersoy, Wireless sensor networks for healthcare: a survey, Computer Networks 54 (2010) 2688-2710.

[15] I.F. Akyildiz, S. Weilian, Y. Sankarasubramaniam, E. Cayirci, A survey on sensor networks, IEEE Communications Magazine 40 (2002) 102114.

[16] IEEE Std 802.15.4, Wireless LAN Medium Access Control (MAC) and Physical Layer (PHY) Specifications for Low-Rate Wireless Personal Area Networks, October 2003.

[17] C. Buratti, A. Conti, D. Dardari, R. Verdone, An overview on wireless sensor networks technology and evolution, Sensors 9 (2009) 68696896.

[18] ZigBee Specification, ZigBee Document 053474r06 Version 1.0, December 2004.

[19] H. Fernandez-Lopez, P. Macedo, J.A. Afonso, J.H. Correia, R. Simões, Evaluation of the impact of the topology and hidden nodes in the performance of a ZigBee network, in: First International Conference on Sensor Systems and Software, Pisa, 2009, pp. 256-271.

[20] H. Fernandez-Lopez, J.A. Afonso, J.H. Correia, R. Simões, Experimental evaluation of IEEE 802.15.4/ZigBee for multi-patient ECG monitoring, in: eHealth2010, Casablanca, 2010, pp. 184-191.

[21] OMNet++ Discrete Event Simulation System. <www.omnetpp.org/>

[22] Jennic Data Sheet JN5139-xxx-Myy JN-DS-JN5139-xxx-Myy 1v6. <http://www.jennic.com/products/modules/jn5139_modules>.

[23] ZigBee Health Care Profile Specification, Revision 15, Version 1.0, March 2010.

[24] L.-J. Chen, T. Sun, N.-C. Liang, An evaluation study of mobility support in ZigBee networks, Journal of Signal Processing Systems 59 (2010) 11

[25] J.H. Kim, B.G. Choi, J. Heo, C.S. Hong, Development of ZigBee Mobile Router for supporting network mobility in healthcare system, in: International Conference on Consumer Electronics, Las Vegas, 2009, pp. 1-2.

[26] F. Cuomo, E. Cipollone, A. Abbagnale, Performance analysis of IEEE 802.15.4 wireless sensor networks: An insight into the topology formation process, Computer Networks 53 (2009) 3057-3075.

[27] Daintree Networks, Sensor Network Analyzer. <http://www. daintree.net/sna/sna.php>.

[28] Daintree Networks, 2400E Sensor Network Adapter. <www. daintree.net/sna/adapter.php>.

[29] H. Karl, A. Willig, Protocols and Architectures for Wireless Sensor Networks, John Wiley \& Sons Ltd., Chichester (UK), 2007.

[30] P. Di Marco, P. Park, C. Fischione, K.H. Johansson, Analytical modelling of IEEE 802.15.4 for multi-hop networks with heterogeneous traffic and hidden terminals, in: IEEE Global Telecommunications Conference, Miami, 2010, pp. 1-6.

[31] G. Bianchi, Performance analysis of the IEEE 802. 11 distributed coordination function, IEEE Journal on Selected Areas in Communications 18 (2000) 535-547.

[32] A. Koubaa, R. Severino, M. Alves, E. Tovar, Improving quality-ofservice in wireless sensor networks by mitigating "hidden-node collisions", IEEE Transactions on Industrial Informatics 5 (2009) 299-313.

[33] H. Lain-Jinn, S. Shiann-Tsong, S. Yun-Yen, C. Yen-Chieh, Grouping strategy for solving hidden node problem in IEEE 802.15.4 LR-WPAN, in: First International Conference on Wireless Internet, Budapest, 2005, pp. 26-32.

[34] A.G. Ruzzelli, R. Tynan, G.M.P. O'Hare, An energy-efficient and lowlatency routing protocol for wireless sensor networks, in: 2005 Systems Communications, Montreal, 2005, pp. 449-454.

[35] J. Mu, K. Liu, A study on the routing selection method in ZigBee networks based on the mobility of nodes and the scale of the network, in: International Conference on Communications and Mobile Computing, Shenzhen, 2010, pp. 405-409.

[36] D. Stevanovic, N. Vlajic, Performance of IEEE 802.15.4 in wireless sensor networks with a mobile sink implementing various mobility strategies, in: $33^{\text {rd }}$ IEEE Conference on Local Computer Networks, Montreal, 2008, pp. 680-688.

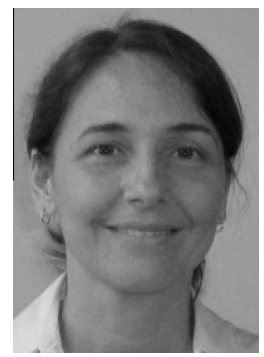

Helena Fernández-López received the Electrical Engineering degree from Rio de Janeiro State University and M.Sc. degree from Rio de Janeiro Federal University, in 1988 and 2005, respectively. She served the Brazilian Navy for 14 years before she moved, in 2005, to Portugal. Since 2007, she is affiliated to University of Minho, as a Ph.D. candidate, in the Engineering Design and Advanced Manufacturing (EDAM) area of the MIT Portugal program. Her main interests are in remote patient monitoring and assisted living technologies.

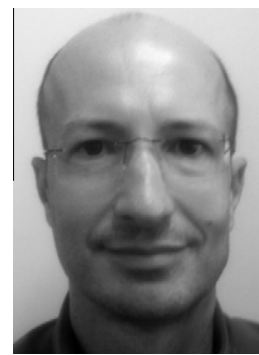

José Augusto Afonso received the Electronic Engineering degree from Universidade Federal do Rio de Janeiro (UFRJ), Brazil, in 1993 the M.Sc. degree in Informatics from Universidade do Minho, Portugal, in 1997, and the Ph.D. degree in Industrial Electronics from Universidade do Minho, in 2005. Currently, he is an Assistant Professor of the Department of Industrial Electronics at Universidade do Minho and member of Centre Algoritmi. His main research interests are in the areas of wireless networks and protocols.

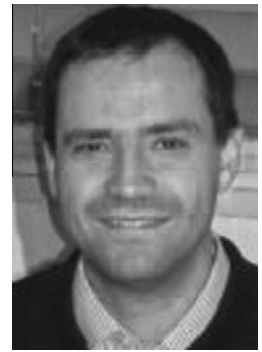

Jose Higino Correia graduated in Physical Engineering from University of Coimbra, Portugal in 1990. He obtained in 1999 a Ph.D. degree at the Laboratory for Electronic Instrumentation, Delft University of Technology, The Netherlands, working in the field of microsystems for optical spectral analysis. Presently, he is a Full Professor in Department of Industrial Electronics, University of Minho, Portugal. He was the General-Chairman of Eurosensors 2003 and MME 2007, Guimarães, Portugal. His professional interests are in micromachining and microfabrication technology for mixed-mode systems, solid-state integrated sensors, microactuators and microsystems.

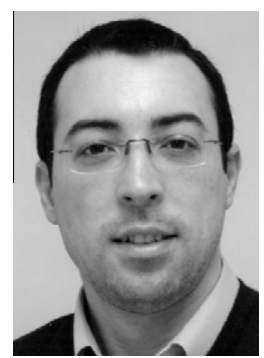

Ricardo Simoes is an Associate Professor at the Polytechnic Institute of Cávado and Ave, Barcelos, Portugal, and a researcher at IPC Institute for Polymers and Composites, University of Minho, Portugal.

He has a 5-year bachelor degree in Polymer Engineering (University of Minho) and a Ph.D. in Materials Science and Engineering (University of North Texas, USA).

He currently coordinates projects in Mobile Health (MIT-Portugal program), Automotive (with Ford Motor Company), Nanomaterials (with US Air Force Research Laboratories), and Medical Devices (with Health Cluster Portugal). He has over 60 scientific publications and made over 20 oral presentations at international conferences. 